Real Analysis Exchange

Vol. 24(2), 1998/9, pp. 677-693

Vittorino Pata, Dipartimento di Matematica, Università di Brescia, 25123

Brescia, Italy. e-mail: pata@bsing.ing.unibs.it

Pietro Ursino, Dipartimento di Matematica, Università di Catania, 95125

Catania, Italy. e-mail: ursino@dipmat.unict.it

\title{
REARRANGEABLE FUNCTIONS ON THE REAL LINE
}

\begin{abstract}
A function $f$ on the real line is rearrangeable if there exists a bijection $\gamma$ on its domain such that $f \circ \gamma$ is continuous. Combinatorial and analytic aspects of the problem are investigated.
\end{abstract}

\section{Introduction}

The problem treated in this paper originates from the idea of giving some meaning to maps between assigned subsets of the real line, when the nature of the coding renders irrelevant the disposition of the points in the space. Translating the concept in a mathematical setting, this means considering, rather than maps, equivalence classes of maps, where the equivalence relation is, for instance, "To have all the corresponding fibers of the same cardinality". In view of this consideration, we decided to privilege, among the representatives, the continuous ones, if there are any. Thus our goal is to "rearrange" a given map, through a permutation of its fibers, in order to get a continuous function (but one might think as well to get the least discontinuous function). The problem is quite vast, and it is purely topological and combinatorial. Indeed, it relies on the existence of continuous functions with a given assignment of the fibers. Many authors have investigated various aspects of this problem (see, e.g., $[2,4,6,7,10]$ and references therein), giving answers to questions as "Does there exist a continuous $k$-to-1 function defined on a given topological space?". Actually, as we will see, the possibility to rearrange a map to a continuous one, depends only on the existence of a continuous function with a given assignment of the cardinality of the fibers. A different point of view is to consider permutations of the fibers that preserve Lebesgue measure. In this case the

Key Words: isomorphisms of measurable sets, rearrangeable functions

Mathematical Reviews subject classification: 26A15, 28D05, 28A20

Received by the editors July 28, 1998 
nature of the problem is analytic. Again, a complete classification of which functions are rearrangeable to continuous ones, through measure preserving permutations of the fibers, seems to be extremely complex. In particular, we dealt with a simple situation, namely, 1-to-1 functions on connected sets. Even in this basic case, though, the analysis is rather delicate.

Notation Throughout the paper, let $\mu$ denote Lebesgue measure on the real line $\mathbb{R}$. We shall use the symbol $|\cdot|$ to stand both for absolute value and for cardinality. Given a set $X$, let $\chi_{X}$ denote the characteristic function of $X$. Finally, let $\mathbb{N}$ denote the set of positive integers.

\section{General Features of Rearrangeable Functions}

We begin introducing the concept of rearrangeable functions. Although for our purposes we consider functions defined on $\mathbb{R}$, the definition could be given, more generally, for functions defined on topological spaces.

Definition 2.1. A function $f: X \subset \mathbb{R} \rightarrow \mathbb{R}$ is said to be rearrangeable to a continuous function (or, more simply, rearrangeable) if there exists a bijective map $\gamma: X \rightarrow X$ such that the function $f \circ \gamma$ is continuous on $X$. Such a $\gamma$ is called a rearrangement of $f$.

Notice that no assumption is made about measurability neither of $f$ nor of $\gamma$.

Let us denote $Y=f(X)$. As an immediate consequence of the definition, if $X$ is connected and/or compact, then $Y$ must be connected and/or compact as well.

In the sequel, we shall assume that $X$ is a connected set, and $Y$ contains more than one point, and consider the set $Y^{X}$; i.e., the set of functions from $X$ onto $Y$. We adopt a classification of the elements of $Y^{X}$ in terms of cardinality of the fibers, which will turn out to be a useful tool to investigate rearrangeability.

Definition 2.2. Introduce the set $\mathbb{A}=\mathbb{N} \cup\left\{\aleph_{0}\right\} \cup\{\mathfrak{c}\}$, and let $\phi: \mathbb{A}^{Y} \rightarrow 2^{Y^{X}}$ be defined by

$$
\phi(f)=\left\{g: g \in Y^{X} \text { such that }\left|g^{-1}(\{y\})\right|=f(y), \forall y \in Y\right\} .
$$

We call $\phi$ a classification of $Y^{X}$ in terms of fibers.

This is a purely combinatorial classification of $Y^{X}$. On the other hand, it discloses a variety of topological problems, which have been widely investigated in recent literature (see, for instance, $[2,4,6,7,10]$ ).

The proof of the following result is trivial. 
Lemma 2.3. Let $\gamma: X \rightarrow X$ be a bijection, and let $f \in Y^{X}$. Then

$$
\left|(f \circ \gamma)^{-1}(\{y\})\right|=\left|f^{-1}(\{y\})\right|, \quad \forall y \in Y .
$$

Observe that, even if the Continuum Hypothesis fails to hold, the absence, of cardinals between $\aleph_{0}$ and $\boldsymbol{c}$ in the set $\mathbb{A}$ is perfectly legitimate by the CantorBendixson theorem. Indeed, if there exists $f \in Y^{X}$ and a rearrangement $\gamma$ of $f$, and $\left|f^{-1}(\{y\})\right|=\alpha$, with $\aleph_{0}<\alpha<\mathfrak{c}$, then, in force of Lemma 2.3, $\left|(f \circ \gamma)^{-1}(\{y\})\right|=\alpha$, which is impossible since $f \circ \gamma$ is a continuous function and $\{y\}$ is a closed set.

The link between rearrangeability and the above classification is expressed in the next proposition.

Proposition 2.4. Let $g \in \phi(f)$ be a continuous function. Then every $h \in$ $\phi(f)$ is rearrangeable.

Proof. We first notice that it is possible to make a decomposition of $X$ in terms of the fibers of any given element of $Y^{X}$. Let $h \in \phi(f)$, and for each $y \in Y$ let $\sigma_{y}$ be any bijection between $h^{-1}(\{y\})$ and $g^{-1}(\{y\})$. Finally, let $\gamma: X \rightarrow X$ be defined as $\gamma(x)=\sigma_{y}(x)$ if $x \in h^{-1}(\{y\})$. It is now apparent that $h \circ \gamma=g$.

Remark 2.5. It is interesting to notice that rearrangeable functions are rather rare. Indeed, if $X, Y \subset \mathbb{R},|Y|=\mathfrak{c}$, then the cardinality of $\mathbb{A}^{Y}$ is $2^{\mathfrak{c}}$, whereas the cardinality of the continuous elements of $Y^{X}$ is at most $\mathfrak{c}$. Thus we conclude that the set of classes of $Y^{X}$ which do not contain any continuous function has cardinality $2^{\mathfrak{c}}$.

As we have already mentioned, the problem of finding a continuous function, with a given assignment of the fibers, has been investigated for the classes $\phi(k)$, with $k \in \mathbb{N}$ (also when $X$ and $Y$ are more general topological space). A classical result in this field is the following [10].

Let $X=Y=[0,1]$ and let $k \in N$, with $k \geq 2$. If $g \in \phi(k)$, then $g$ must have infinitely many discontinuities.

More generally, the same result holds if $X$ is a continuum and $Y$ is a dendrite [7].

Concerning the case of fibers of infinite cardinality, we show that the class $\phi\left(\aleph_{0}\right)$ has a continuous element. The following is a slightly different variant of an example due to Ciesielski. Let $\mathcal{V}:[0,1] \rightarrow[0,1]$ be the Cantor-Vitali function (see, e.g., [8] for the definition), and denote the Cantor ternary set by $T$. Then $[0,1] \backslash T=\bigcup_{n \in \mathbb{N}}\left(a_{n}, b_{n}\right)$ (disjoint union). Setting $p=\log 2 / \log 3$, 
the function

$$
f(x)=\mathcal{V}(x) \chi_{T}(x)+\sum_{n \in \mathbb{N}}\left(\mathcal{V}\left(a_{n}\right)+\left(b_{n}-a_{n}\right)^{p} \sin \left(2 \pi \frac{x-a_{n}}{b_{n}-a_{n}}\right)\right) \chi_{\left(a_{n}, b_{n}\right)}(x)
$$

is easily seen to fulfill our requirements (see also [2], Proposition 1.15).

Finally, the class $\phi(\mathfrak{c})$ possesses also a continuous element. Indeed, it is well-known that there exists a function $\mathcal{P}:[0,1] \rightarrow[0,1] \times[0,1]$ onto (the Peano function). Thus the function $\Pi_{1} \circ \mathcal{P}$, where $\Pi_{1}$ is projection of the unit square on the first component, serves our purposes. The space $X$ can be replaced by a locally connected continuum. (Recall that a continuum is a compact connected metric space.) In this case there exists a continuous surjection from $X$ onto its square. In general, this continuous surjection does not exist if $X$ is a continuum which is not locally connected (see [9]), and therefore it is not clear if there exists a continuous $\mathbf{c}$-to- 1 function on such $X$.

The situation is quite different if $X \subset \mathbb{R}$ is an open connected set. Since $X$ is homeomorphic to $\mathbb{R}$, we can (and do) assume $X=\mathbb{R}$. Extending the function $\Pi_{1} \circ \mathcal{P}$ on $\mathbb{R}$, it is readily seen that there exists a continuous $\mathfrak{c}$-to- 1 function on $\mathbb{R}$. Moreover, the function $f(x)=x \sin x$ is continuous and $\aleph_{0^{-}}$ to-1. For the case of fibers of finite cardinality, it is quite easy to show that no continuous function $\alpha$-to- 1 exists for $\alpha$ even, whereas it is possible to build examples of continuous functions $\alpha$-to- 1 for any $\alpha$ odd (see [2], Proposition 1.2).

By means of of Proposition 2.4, we summarize the considerations about functions with all the fibers of the same cardinality in the next theorem.

Theorem 2.6. Let $f: X \subset \mathbb{R} \rightarrow \mathbb{R}$ be such that $f \in \phi(\alpha)$, with $\alpha \in \mathbb{A}$.

(i) If $X$ is a connected compact set, then $f$ is rearrangeable if and only if $\alpha \in\left\{1, \aleph_{0}, \mathbf{c}\right\}$.

(ii) If $X$ is a connected open set, then $f$ is rearrangeable if and only if $\alpha \neq 2 k$, $k \in \mathbb{N}$.

The above discussion is limited to the case of functions with all the fibers of the same cardinality. It would also be interesting to consider some classes of functions whose fibers are not of the same cardinality. This problem has been recently investigated in [2] (and completely solved for functions with finite fibers).

We conclude this section mentioning that rearrangeability and measurability are not related concepts. Using Proposition 2.4, it is possible to construct 
an example of a rearrangeable function which is not measurable. For instance, let $P \subset \mathbb{R}$ be a nonmeasurable set, with $|\mathbb{R} \backslash P|=\mathfrak{c}$, and consider the function

$$
f(x)= \begin{cases}0 & \text { if } x \in P, \\ \sigma(x) & \text { if } x \in \mathbb{R} \backslash P,\end{cases}
$$

where $\sigma$ is any bijection from $P$ onto $\mathbb{R} \backslash\{0\}$.

Conversely, in Example 3.9 below, we construct a measurable function which is not rearrangeable.

\section{Isomorphisms of Measurable Sets}

The aim of this section is to introduce measure preserving bijective maps between measurable sets, and discuss some related results. The idea was first envisaged by Rohlin [12].

Definition 3.1. Let $X, Y \subset \mathbb{R}$ be measurable sets. A bijective map $\gamma: X \rightarrow$ $Y$ is said to be an isomorphism between $X$ and $Y$ if for every measurable set $M \subset Y$ the set $\gamma^{-1}(M)$ is measurable, and

$$
\mu\left(\gamma^{-1}(M)\right)=\mu(M)
$$

Thus, in particular, $\mu(X)=\mu(Y)$. If $X$ and $Y$ coincide, such a $\gamma$ is said to be an endomorphism.

Remark 3.2. It is clear from the definition that if $\gamma_{1}$ is an isomorphism between $X$ and $Y$, and $\gamma_{2}$ is an isomorphism between $Y$ and $Z$, then $\gamma_{2} \circ \gamma_{1}$ is an isomorphism between $X$ and $Z$.

We will use the following result due to Rohlin (see [12], p.20).

Lemma 3.3. Let $X \subset \mathbb{R}$ be a measurable set, and let $\gamma: X \rightarrow \mathbb{R}$ be an injective map such that $\gamma(X)$ is measurable. Assume that $\gamma^{-1}(M)$ is measurable, and $\mu\left(\left(\gamma^{-1}(M)\right)=\mu(M)\right.$, for every measurable set $M \subset \gamma(X)$. Then $\gamma$ carries measurable sets into measurable sets.

The next proposition is an easy consequence of the above lemma. For the reader's convenience we give an outline of the proof.

Proposition 3.4. Let $X, Y \subset \mathbb{R}$ be measurable sets, and let $\gamma: X \rightarrow Y$ be a one-to-one and onto mapping. The following are equivalent.

(i) For every $O \subset Y, O$ open in $Y$, the set $\gamma^{-1}(O)$ is measurable, and $\mu\left(\gamma^{-1}(O)\right)=\mu(O)$. 
(ii) $\gamma$ is an isomorphism between $X$ and $Y$.

(iii) $\gamma$ is an isomorphism between $X$ and $Y$, and $\gamma^{-1}$ is an isomorphism between $Y$ and $X$.

Proof. Clearly, (iii) implies (ii), which in turn implies (i). We show that (i) implies (iii). We first suppose that $\mu(X)=\mu(Y)=L<\infty$. From (i), $\gamma$ is measurable. Moreover for every set $C \subset Y, C$ closed in $Y$,

$$
\mu\left(\gamma^{-1}(C)\right)=L-\mu\left(\gamma^{-1}(Y \backslash C)\right)=L-\mu(Y \backslash C)=\mu(C) .
$$

Let now $M \subset Y$ be a measurable set. Then, given any $\varepsilon>0$, there exist an open set $O$ and a closed set $C$ (open and closed in $Y$ ) such that $C \subset M \subset$ $O \subset Y$, and $\mu(O \backslash C)<\varepsilon$. Thus

$$
\mu(C)=\mu\left(\gamma^{-1}(C)\right) \leq \mu_{*}\left(\left(\gamma^{-1}(M)\right) \leq \mu^{*}\left(\left(\gamma^{-1}(M)\right) \leq \mu\left(\gamma^{-1}(O)\right)=\mu(O),\right.\right.
$$

where $\mu_{*}$ and $\mu^{*}$ denote Lebesgue inner measure and outer measure, respectively. Recall that, for any subset $F \subset \mathbb{R}$

$$
\begin{aligned}
& \mu_{*}(F)=\sup \{\mu(K): K \subset F, K \text { compact }\} \\
& \mu^{*}(F)=\inf \{\mu(O): O \supset F, O \text { open }\} .
\end{aligned}
$$

Therefore, due to the arbitrariness of $\varepsilon$, we conclude that $\gamma^{-1}(M)$ is measurable, and $\mu\left(\left(\gamma^{-1}(M)\right)=\mu(M)\right.$. According to the Lemma 3.3, $\gamma$ carries measurable sets into measurable sets. Finally, let $N \subset X$ be measurable. Then $M=\gamma(N)$ is measurable as well, and

$$
\mu(\gamma(N))=\mu(M)=\mu\left(\gamma^{-1}(M)\right)=\mu(N) .
$$

If $\mu(X)=\mu(Y)=\infty$, for every $n \in \mathbb{Z}$ define $Y_{n}=Y \cap[n, n+1)$, and let $X_{n}=\gamma^{-1}\left(Y_{n}\right)$. The restriction of $\gamma$ mapping $X_{n}$ to $Y_{n}$ satisfies condition (i) of the proposition, and thus, by the above proof, also fulfills (iii). It is then readily seen that (iii) holds.

The next theorem, needed in the course of the investigation, is known. However we were not able to locate a proof, so that here we provide a quite simple one ${ }^{1}$.

Theorem 3.5. Let $X, Y \subset \mathbb{R}$ be two measurable sets having the same positive measure. Then there exists an isomorphism $\gamma$ between $X$ and $Y$.

\footnotetext{
${ }^{1}$ After we finished the paper we learned of a proof contained in the treatise [3].
} 
Proof. Firstly, notice that if $\beta$ is an isomorphism between two sets $A$ and $B$ of finite positive measure, it is possible to build an isomorphism $\gamma$ between $A \cup N$ and $B \cup R$, where $N$ and $R$ are two any sets of measure zero. Upon substituting $N$ with $N \backslash A$, and $R$ with $R \backslash B$, we may assume that the above unions are disjoint. Remove an uncountable null set from $A$ and let $R_{0}=\beta\left(N_{0}\right)$. Since $\beta$ is an isomorphism, $R_{0}$ is an uncountable null set. Put $N_{1}=N_{0} \cup N$ and $R_{1}=R_{0} \cup R$, and let $\sigma$ be any bijective map from $N_{1}$ to $R_{1}$. Finally, define the bijective map $\gamma: A \cup N \rightarrow B \cup R$ by

$$
\gamma(x)= \begin{cases}\beta(x) & \text { if } x \in A \backslash N_{0}, \\ \sigma(x) & \text { if } x \in N_{1} .\end{cases}
$$

It is then apparent that such a $\gamma$ is an isomorphism.

Assume first that $X$ is a bounded set, say, $X \subset[-K, K]$ for some $K>0$, with $\mu(X)=L$. In view of Remark 3.2 and Proposition 3.4, it is enough to prove the existence of an isomorphism between $X$ and the interval $[0, L]$. Introduce the function $\varphi:[-K, K] \rightarrow[0, L]$ by

$$
\varphi(x)=\int_{-K}^{x} \chi_{X}(y) d y .
$$

Since $\varphi$ is absolutely continuous, it maps measurable sets into measurable sets, and null sets into null sets. Let $\left\{x_{n}\right\}_{n \in \mathbb{N}}$ be a dense countable set contained in $X$. For every $n \in \mathbb{N}$, set

$$
A_{n}=\left\{x \in X, x \neq x_{n} \text { such that } \varphi(x)=\varphi\left(x_{n}\right)\right\} .
$$

Then $\mu\left(A_{n}\right)=\mu\left(\varphi\left(A_{n}\right)\right)=0$. Furthermore, let

$$
B=\{x \in X \text { such that }(x, x+\varepsilon) \cap X=\emptyset \text { for some } \varepsilon>0\} .
$$

The set $B$ is at most countable. Putting $N=\bigcup_{n \in \mathbb{N}} A_{n} \cup B$, it is clear that $\mu(N)=0$. It is now easy to see that $\varphi_{\mid X \backslash N}$ is one-to-one. Indeed, given $x<y$ in $X$ with $\varphi(x)=\varphi(y)$, either $x \in B$, or there exists some $x_{n_{0}} \in[x, y]$. In that case, since $\varphi$ is increasing, $\varphi\left(x_{n_{0}}\right)=\varphi(y)$, which implies that $y \in A_{n_{0}}$. We now claim that

$$
\mu(O \cap(X \backslash N))=\mu(\varphi(O \cap(X \backslash N)))
$$

for every open set $O \subset[-K, K]$, which is equivalent to proving that

$$
\mu(O \cap X)=\mu(\varphi(O \cap X)) .
$$


Since an open set is a disjoint union of open intervals, it is enough to verify the claim true for $O=(a, b) \subset[-K, K]$. Exploiting the fact that $\varphi$ is continuous and increasing, set

$$
x^{+}=\max \{x \geq b \text { such that } \varphi(x)=\varphi(b)\}
$$

and

$$
x^{-}=\min \{x \leq a \text { such that } \varphi(x)=\varphi(a)\} .
$$

It is then straightforward to see that

$$
\mu\left(X \cap\left[b, x^{+}\right]\right)=0 \quad \text { and } \quad \mu\left(X \cap\left[x^{-}, a\right]\right)=0 .
$$

We claim that

$$
x \in X \text { and } \varphi(x) \in \varphi([a, b] \cap X)
$$

if and only if

$$
x \in X \cap\left((a, b) \cup\left[x^{-}, a\right] \cup\left[b, x^{+}\right]\right) .
$$

Indeed, since $\varphi$ is increasing, if $x \in X$ and $\varphi(x) \in \varphi([a, b] \cap X)$ it must necessarily be that $x \in O \cup\left[x^{-}, a\right] \cup\left[b, x^{+}\right]$. Since $X \cap\left(\left[x^{-}, a\right] \cup\left[b, x^{+}\right]\right)$has null measure, we conclude that

$$
\chi_{X}(x) \chi_{\varphi([a, b] \cap X)}(\varphi(x))=\chi_{(a, b) \cap X}(x) \quad \text { a.e. }(\mu) .
$$

Thus, using a change of variable (see, for instance, [8]), we get

$$
\begin{aligned}
\mu((a, b) \cap X) & =\int_{-K}^{K} \chi_{(a, b) \cap X}(y) d y=\int_{-K}^{K} \chi_{X}(y) \chi_{\varphi((a, b) \cap X)}(\varphi(y)) d y \\
& =\int_{-K}^{K} \varphi^{\prime}(y) \chi_{\varphi((a, b) \cap X)}(\varphi(y)) d y=\int_{0}^{L} \chi_{\varphi((a, b) \cap X)}(y) d y \\
& =\mu(\varphi((a, b) \cap X)),
\end{aligned}
$$

as claimed. Hence, calling $Y=\varphi(X \backslash N)$, from Proposition 3.4 applied to $\varphi_{\mid X \backslash N}^{-1}$ we conclude that $\varphi_{\mid X \backslash N}$ is an isomorphism between $X \backslash N$ and $Y$, so, in particular, $\mu(Y)=\mu(X \backslash N)=L$; i.e., $[0, L]=Y \cup R$, with $\mu(R)=0$. Recalling the first part of the proof, we get the desired isomorphism between $X$ and $[0, L]$.

Suppose now that $X$ is not a bounded set, with $\mu(X)=L$. Again, by Remark 3.2 and Proposition 3.4, it is enough to show that there exists an isomorphism between $X$ and the interval $[0, L)\left(\mathbb{R}^{+}\right.$if $\left.L=\infty\right)$. Write $X=$ $\bigcup_{n \in I} X_{n}, I=\{1,2, \ldots, N\}$, with $N$ possibly infinite, where the $X_{n}$ 's are bounded and pairwise disjoint subsets of $\mathbb{R}$ of positive measure $J_{n}$. Thus 
for every $n \in I$ it is possible to find an isomorphism $\gamma_{n}$ between $X_{n}$ and $\left[0, J_{n}\right)$. Defining then $L_{1}=0$, and $L_{n}=\sum_{k=1}^{n-1} J_{k}$ for $n \geq 1$ (notice that $L_{n} \rightarrow L$ ), the map $\beta_{n}=\gamma_{n}+L_{n}$ defined on $X_{n}$ is an isomorphism between $X_{n}$ and $\left[L_{n}, L_{n}+J_{n}\right)$, and therefore the map $\gamma(x)=\beta_{n}(x)$, if $x \in X_{n}$, is an isomorphism between $X$ and $[0, L)$.

\section{Completely Rearrangeable Functions}

So far, we treated rearrangeability of functions only from a set theoretical point of view. In this section we let measure theory enter the picture, and we focus our attention on measure preserving rearrangements. We begin with a definition.

Definition 4.1. A real-valued function $f$ defined on a measurable set $X \subset \mathbb{R}$ is said to be completely rearrangeable to a continuous function (or, more simply, completely rearrangeable) if there exists an endomorphism $\gamma$ of $X$ such that the function $f \circ \gamma$ is continuous on $X$. Such a $\gamma$ is called a complete rearrangement of $f$.

Remark 4.2. It is quite immediate that a completely rearrangeable function $f: X \subset \mathbb{R} \rightarrow \mathbb{R}$ is measurable on $X$. Indeed, let $O \subset \mathbb{R}$ be an open set. Then $\gamma^{-1}\left(f^{-1}(O)\right)$ is open, and since $\gamma^{-1}$ carries nonmeasurable sets into nonmeasurable sets, we get that $f^{-1}(O)$ is measurable.

A direct application of the Lebesgue monotone and dominated convergence theorems yields the following proposition.

Proposition 4.3. Let $f: X \subset \mathbb{R} \rightarrow \mathbb{R}$ be completely rearrangeable, with rearrangement $\gamma$. Then $f \in L^{1}(X)$ if and only if $f \circ \gamma \in L^{1}(X)$. In that case

$$
\int_{X} f(x) d x=\int_{X} f \circ \gamma(x) d x .
$$

Indeed, the above result clearly holds when $f$ is a simple function. In the general case, there exists a sequence $s_{n}$ of simple functions such that $s_{n} \rightarrow f$ and $0 \leq\left|s_{1}\right| \leq\left|s_{2}\right| \leq \cdots \leq|f|$ almost everywhere.

It would be interesting now to exhibit necessary and sufficient conditions for a function in order to be completely rearrangeable. Obviously, necessary conditions for rearrangeability are also necessary for complete rearrangeability. Also, it is reasonable to restrict our attention to the case of functions defined on a connected subset $X$ of the real line. However, a general criterion to establish which functions defined on $X$ are completely rearrangeable does not 
seem to be easily detectable. Here we consider the simplest possible case; i.e., one-to-one measurable functions. We begin by investigating the particular case when $X$ is a closed interval.

Lemma 4.4. Let $X \subset \mathbb{R}$ be a closed bounded interval, and let $f: X \rightarrow \mathbb{R}$ be a one-to-one measurable function. Then $f$ is completely rearrangeable if and only if $f(X)$ is a closed interval, and

$$
\mu\left(f^{-1}([a, b])\right)>0, \quad \forall a, b \in f(X) \text { s.t. } a<b .
$$

Proof. If $f$ is completely rearrangeable, then there exists an endomorphism $\gamma$ of $X$ such that $\Psi=f \circ \gamma$ is continuous. Clearly, $f(X)=\Psi(X)$, and therefore $f(X)$ is compact and connected. Moreover, since $\Psi$ is continuous on $X$, it must be either strictly increasing or strictly decreasing. Let $a, b \in f(X)$ such that $a<b$. Then

$$
\mu\left(f^{-1}([a, b])\right)=\mu\left(\gamma\left(\Psi^{-1}([a, b])\right)\right)=\mu\left(\Psi^{-1}([a, b])\right)=\left|\Psi^{-1}(b)-\Psi^{-1}(a)\right|>0 .
$$

Conversely, suppose that $f(X)$ is a closed interval and that $(\dagger)$ holds. There is no loss of generality in assuming $X=f(X)=[0,1]$. Select $n \in \mathbb{N}$, and let

$$
0=a_{0}^{n}<a_{1}^{n}<\cdots<a_{2^{n}}^{n}=1
$$

be such that the sets

$$
A_{j}^{n}=f^{-1}\left(\left[a_{j-1}^{n}, a_{j}^{n}\right)\right), \quad j=1, \ldots, 2^{n}-1
$$

and

$$
A_{2^{n}}^{n}=f^{-1}\left(\left[a_{2^{n}-1}^{n}, a_{2^{n}}^{n}\right]\right)
$$

LL have measure $1 / 2^{n}$. Since $\mu\left(f^{-1}(\{x\})\right)=0$ for every $x \in[0,1]$, it is wellknown that $\mu\left(f^{-1}([0, x])\right)$ is a continuous and increasing function onto $[0,1]$ (see, e.g., [1]). Therefore the $a_{j}^{n}$ 's are well defined and unique. Finally, define the intervals

$$
I_{j}^{n}=\left[(j-1) / 2^{n}, j / 2^{n}\right), \quad j=1, \ldots, 2^{n}-1
$$

and

$$
I_{2^{n}}^{n}=\left[\left(2^{n}-1\right) / 2^{n}, 1\right] .
$$

By virtue of Theorem 3.5, for every $j$ there exists an isomorphism $\gamma_{j}^{n}$ between $A_{j}^{n}$ and $I_{j}^{n}$. Thus the map $\gamma_{n}:[0,1] \rightarrow[0,1]$ equal to $\gamma_{j}^{n}$ on $A_{j}^{n}$ is an endomorphism of $[0,1]$. We introduce two sequences of functions; namely $f_{n}(x)=\sum_{j=1}^{2^{n}} a_{j-1}^{n} \chi_{A_{j}^{n}}(x)$, and $\Psi_{n}(x)=f_{n} \circ \gamma_{n}^{-1}(x)$. The proof will be completed through several steps. 
StEP 1. For every $m, n, p \in \mathbb{N}$, with $n=m+p$, we have

$$
a_{j}^{m}=a_{j 2^{p}}^{n}, \quad \forall j=0,1, \ldots, 2^{m} .
$$

Moreover if $A_{j}^{m} \cap A_{i}^{n} \neq 0$, then $A_{i}^{n} \subset A_{j}^{m}$.

The quite easy proof of this step, which relies upon combinatorial arguments, is left to the reader.

Step 2. For every $n \in \mathbb{N}$, set

$$
K_{n}=\max \left\{a_{j+1}^{n}-a_{j}^{n}: j=0,1, \ldots, 2^{n}-1\right\} .
$$

Then $\lim _{n \rightarrow \infty} K_{n}=0$.

It is clear that $K_{n}$ is decreasing. Assume that $K_{n} \rightarrow K>0$, and let $0<C<$ $K$. Since $K_{n} \geq K>C$, for every $n \in \mathbb{N}$, there exists a closed interval $J_{n}$ of length $C$ such that $J_{n}$ does not contain any $a_{j}^{n}$. Take a partition of $[0,1]$ of $s$ pairwise disjoint (except for the endpoints) closed intervals of equal length $L=C / 2$, denoted by $B_{1}, \ldots, B_{s}$. For every $k=1, \ldots, s$, let $N_{k}$ be the number of intervals $J_{n}$ containing $B_{k}$. Notice that every $J_{n}$ must contain a $B_{k}$. Since the $J_{n}$ 's are infinite, at least one of the $N_{k}$ 's must be infinite. Therefore there exists an interval of length $L$ corresponding to some $k$, which we call $B$ for simplicity, and a subsequence $J_{n_{l}}$ of intervals such that $B \subset \bigcap J_{n_{l}}$. Hence $B$ does not contain any $a_{j}^{n_{l}}$. But $n_{l} \rightarrow \infty$, and by Step 1 we conclude that $B$ does not contain any $a_{j}^{n}$ either. Writing $B=[\alpha, \beta]$ (We can assume $\alpha>0$ and $\beta<1$.), for every $n \in \mathbb{N}$ let $j_{n}$ be such that $a_{j_{n}-1}^{n}<\alpha<\beta<a_{j_{n}}^{n}$. Then

$$
\mu\left(f^{-1}([\alpha, \beta]) \leq \limsup _{n \rightarrow \infty} \mu\left(A_{j_{n}}^{n}\right)=\limsup _{n \rightarrow \infty} \frac{1}{2^{n}}=0,\right.
$$

contrary to $(\dagger)$.

STEP 3. $\gamma_{n} \rightarrow \gamma$ uniformly on $[0,1]$, where $\gamma$ is an isomorphism between $[0,1]$ and $[0,1] \backslash N$, being $N$ a null set (possibly empty).

We first show that $\gamma_{n}$ is a Cauchy sequence in the topology of uniform convergence. Fix $n_{0} \in \mathbb{N}$, and let $n, m \geq n_{0}$. Set $x \in[0,1]$. Then $x \in A_{i}^{n} \cap A_{k}^{m}$ for some $i, k$, but also $x \in A_{j}^{n_{0}}$ for some $j$. By Step $1, A_{i}^{n} \cap A_{k}^{m} \subset A_{j}^{n_{0}}$, and the same inclusion is fulfilled by the corresponding sets $I_{i}^{n}, I_{k}^{m}, I_{j}^{n_{0}}$. Thus $\gamma_{n}(x), \gamma_{m}(x) \in I_{j}^{n_{0}}$, and we conclude that $\left|\gamma_{n}(x)-\gamma_{m}(x)\right| \leq \frac{1}{2^{n_{0}}}$. The above relation holds for every $x$, which yields the desired conclusion. Hence there exists $\gamma$ such that $\gamma_{n} \rightarrow \gamma$ uniformly on [0,1]. In particular, $\gamma$ is measurable. We now show that $\gamma$ is one-to-one. Indeed, let $x \neq y$. Since $f(x) \neq f(y)$, from Step 2 we get that for $n_{0}$ big enough, between $x$ and $y$ there are at least two $a_{j}^{n_{0}}$ 's. Thus, assuming $f(x)<f(y), x \in A_{j}^{n_{0}}$ and $y \in A_{i}^{n_{0}}$, with $i \geq j+2$. It follows that $\gamma_{n_{0}}(x) \in I_{j}^{n_{0}}$ and $\gamma_{n_{0}}(y) \in I_{i}^{n_{0}}$, which yields $\left|\gamma_{n_{0}}(x)-\gamma_{n_{0}}(y)\right|>\frac{1}{2^{n_{0}}}$. 
Notice that if $n \geq n_{0}$, then $x \in A_{l}^{n} \subset A_{j}^{n_{0}}$ and $y \in A_{s}^{n} \subset A_{i}^{n_{0}}$, for some $l, s$. Thus $\gamma_{n}(x) \in I_{l}^{n} \subset I_{j}^{n_{0}}$ and $\gamma_{n_{0}}(y) \in I_{s}^{n} \subset I_{i}^{n_{0}}$, and the above inequality holds for $n$ as well, and therefore it holds for the limit. In order to complete the step, choose $0 \leq a<b \leq 1$. Let $0<\varepsilon<b-a$, and let $n$ be such that

$$
\left\|\gamma_{n}-\gamma\right\|_{\infty}=\sup _{x \in[0,1]}\left|\gamma_{n}(x)-\gamma(x)\right|<\varepsilon .
$$

Then the inclusion

$$
\gamma_{n}^{-1}((a+\varepsilon, b-\varepsilon)) \subset \gamma^{-1}((a, b)) \subset \gamma_{n}^{-1}((a-\varepsilon, b+\varepsilon))
$$

holds. Hence $b-a-2 \varepsilon \leq \mu\left(\gamma^{-1}((a, b))\right) \leq b-a+2 \varepsilon$. From the arbitrariness of $\varepsilon, \mu\left(\gamma^{-1}((a, b))\right)=b-a$. Let now $O$ be an open set in $[0,1]$. Then $O$ is a countable union of pairwise disjoint open intervals $O_{i}$. Due to the injectivity of $\gamma$,

$$
\mu\left(\gamma^{-1}(O)\right)=\mu\left(\gamma^{-1}\left(\bigcup_{i} O_{i}\right)\right)=\sum_{i} \mu\left(\gamma^{-1}\left(O_{i}\right)\right)=\mu\left(\bigcup_{i} O_{i}\right)=\mu(O) .
$$

From Proposition 3.4 we deduce that $\gamma$ is an isomorphism between $[0,1]$ and $\gamma([0,1])$. Clearly, $\gamma([0,1]) \subset[0,1]$. In particular, $\mu(\gamma([0,1]))=1$; i.e., $N=$ $[0,1] \backslash \gamma([0,1])$ is a nullset.

STEP 4. $f_{n}(x) \rightarrow f(x)$ for every $x \in[0,1]$.

Let $x \in[0,1]$, and select $\varepsilon>0$. From Step 2, for $n_{0}$ big enough, we have that $K_{n}<\varepsilon$ for $n \geq n_{0}$. Fix then $n \geq n_{0}$, and let $a_{j_{0}}^{n}$ the biggest $a_{j}^{n}$ less than or equal to $f(x)$. By construction of $f_{n}$, we get that $f_{n}(x)=a_{j_{0}}^{n}$. Thus

$$
\left|f(x)-f_{n}(x)\right|=f(x)-a_{j_{0}}^{n}<a_{j_{0}+1}^{n}-a_{j_{0}}^{n} \leq K_{n}<\varepsilon .
$$

STEP $5 . \quad \Psi_{n} \rightarrow \Psi$ uniformly on $[0,1]$, where $\Psi$ is a continuous, strictly increasing function onto $[0,1]$.

We first show that $\Psi_{n}$ is a Cauchy sequence in the topology of the uniform convergence. Fix $n_{0} \in \mathbb{N}$, and let $n, m \geq n_{0}$. Set $x \in[0,1]$, and assume that $n=m+p$. Then $x \in I_{i}^{n} \subset I_{k}^{m}$ for some $i, k$, and therefore $\gamma_{n}^{-1}(x) \in A_{i}^{n}$ and $\gamma_{m}^{-1}(x) \in A_{k}^{m}$. From Step $1, i \in\left\{(k-1) 2^{p}+1, \ldots, k 2^{p}\right\}$; so $\Psi_{n}(x)=a_{i-1}^{n}$ and $\Psi_{m}(x)=a_{k-1}^{m}$, with $a_{i-1}^{n} \in\left[a_{k-1}^{m}, a_{k}^{m}\right]$. Hence

$$
\left|\Psi_{n}(x)-\Psi_{m}(x)\right|=a_{i-1}^{n}-a_{k-1}^{m} \leq a_{k}^{m}-a_{k-1}^{m} \leq K_{m} \leq K_{n_{0}},
$$

and the conclusion follows from Step 2, since $n_{0}$ is independent of $x$. Thus $\Psi_{n}$ converges to some $\Psi$ uniformly on $[0,1]$. Since $[0,1]$ is closed, $\Psi([0,1]) \subset[0,1]$. Moreover $\Psi$ is increasing, being the limit of increasing functions. We show 
that $\Psi$ is strictly increasing. Indeed, let $x \neq y$, and let $n_{0}$ be so large that $x \in I_{j}^{n_{0}}$ and $y \in I_{i}^{n_{0}}$, with $i \geq j+2$. Thus $\Psi_{n_{0}}(x)=a_{j-1}^{n_{0}}$ and $\Psi_{n_{0}}(y)=a_{i-1}^{n_{0}}$, which implies

$$
\left|\Psi_{n_{0}}(x)-\Psi_{n_{0}}(y)\right|>a_{i-1}^{n_{0}}-a_{j}^{n_{0}} \geq a_{j+1}^{n_{0}}-a_{j}^{n_{0}}>0
$$

If $n \geq n_{0}$, then $\Psi_{n}(x)=a_{l-1}^{n}$ and $\Psi_{n}(y)=a_{k-1}^{n}$, and since $\gamma_{n}^{-1}(x) \in A_{l}^{n} \subset A_{j}^{n_{0}}$ and $\gamma_{n}^{-1}(y) \in A_{k}^{n} \subset A_{i}^{n_{0}}$, from Step 1 we get that $a_{l-1}^{n}<a_{j}^{n_{0}}$ and $a_{k-1}^{n}>a_{i-1}^{n_{0}}$. Thus the above inequality holds for $n$ as well, and therefore it holds to the limit. From the construction of the $\Psi_{n}$ 's, and by Step 2 ,

$$
\Psi(0)=\lim _{n \rightarrow \infty} \Psi_{n}(0)=0, \quad \text { and } \quad \Psi(1)=\lim _{n \rightarrow \infty} \Psi_{n}(1)=\lim _{n \rightarrow \infty} a_{2^{n}-1}^{n}=1 .
$$

We are left to prove the continuity of $\Psi$, which, together with the strict monotonicity and the fact that $\Psi(0)=0$ and $\Psi(1)=1$, implies that $\Psi$ is onto. Notice first that $\Psi_{n}$ is right-continuous, and thus $\Psi$ is right continuous too. Assume that there exists a point of (left) discontinuity $x \in(0,1]$. Then, for some $R>0, \Psi(x)-\Psi(y) \geq R, \quad \forall y<x$. Choose $n$ so large that $\left\|\Psi_{n}-\Psi\right\|_{\infty}<$ $R / 3$ and $K_{n}<R / 3$ (again, we use Step 2). For some $j$, we have $x \in I_{j}^{n}$. Select $y<x$ so close to $x$ that $y \in I_{j-1}^{n} \cup I_{j}^{n}$. We get

$$
\begin{aligned}
\Psi(x)-\Psi(y) & \leq\left|\Psi(x)-\Psi_{n}(x)\right|+\Psi_{n}(x)-\Psi_{n}(y)+\left|\Psi(y)-\Psi_{n}(y)\right| \\
& \leq 2\left\|\Psi_{n}-\Psi\right\|_{\infty}+a_{j-1}^{n}-a_{j-2}^{n} \\
& \leq 2 R / 3+K_{n}<R,
\end{aligned}
$$

which is inconsistent with the inequality $\Psi(x)-\Psi(y) \geq R, \quad \forall y<x$.

We now have the tools to complete the proof. Since $\Psi$ is continuous on $[0,1], \Psi$ is uniformly continuous. Choose $\varepsilon>0$, and let $\delta=\delta(\varepsilon)$ such that $|x-y|<\delta$ implies $|\Psi(x)-\Psi(y)|<\varepsilon$. Let $n_{0}$ be such that $\left\|\Psi_{n}-\Psi\right\|_{\infty}<\varepsilon$ and $\left\|\gamma_{n}-\gamma\right\|_{\infty}<\delta$ for every $n \geq n_{0}$. Fix $x \in[0,1]$. Then for $n \geq n_{0}$

$$
\begin{aligned}
\left|\Psi_{n} \circ \gamma_{n}(x)-\Psi \circ \gamma(x)\right| & \leq\left|\Psi_{n} \circ \gamma_{n}(x)-\Psi \circ \gamma_{n}(x)\right|+\left|\Psi \circ \gamma_{n}(x)-\Psi \circ \gamma(x)\right| \\
& \leq\left\|\Psi_{n}-\Psi\right\|_{\infty}+\left|\Psi\left(\gamma_{n}(x)\right)-\Psi(\gamma(x))\right|<2 \varepsilon .
\end{aligned}
$$

Since $n_{0}$ is independent of $x$, we conclude that $\Psi_{n} \circ \gamma_{n} \rightarrow \Psi \circ \gamma$ uniformly on $[0,1]$. On the other hand, $\Psi_{n} \circ \gamma_{n}=f_{n}$, and, since $f_{n} \rightarrow f$, we have that $f=$ $\Psi \circ \gamma$. Notice that $f$ and $\Psi$ are bijections from $[0,1]$ to $[0,1]$. Hence $\gamma=\Psi^{-1} \circ f$ is a bijection too, which means that $N=\emptyset$. Thus from Proposition 3.4, $\gamma^{-1}$ is an endomorphism of $[0,1]$, and $f \circ \gamma^{-1}=\Psi$, as desired.

It is apparent that there are only two possible rearranged continuous functions coming from $f$, precisely the function $\Psi$ of the lemma and the function 
$\Phi=1-\Psi$, which is strictly decreasing, corresponding to the rearrangement $\gamma \circ \tilde{\gamma}$, with $\tilde{\gamma}(x)=1-x$. Indeed, if $\Psi_{1}$ and $\Psi_{2}$ are two increasing rearrangements of $f$, for every $x \in[0,1]$ we have

$$
\Psi_{1}^{-1}(x)=\mu\left(\Psi_{1}^{-1}([0, x])\right)=\mu\left(\Psi_{2}^{-1}([0, x])\right)=\Psi_{2}^{-1}(x) .
$$

Corollary 4.5. Let $X \subset \mathbb{R}$ be an open (resp. half-open) bounded interval, and let $f: X \rightarrow \mathbb{R}$ be a one-to-one measurable function. Then $f$ is completely rearrangeable if and only if $f(X)$ is an open (resp. half-open) bounded interval, and $(\dagger)$ holds.

Proof. Consider the case $X=(a, b)$, the other being analogous. Necessity is proved as in Lemma 4.4. (Here we use the fact that $f$ is 1-to-1.) To prove sufficiency, setting $f(I)=(c, d)$, define

$$
\tilde{f}(x)= \begin{cases}c & \text { if } x=a, \\ f(x) & \text { if } x \in(a, b), \\ d & \text { if } x=b\end{cases}
$$

From Lemma 4.4 we conclude that there exists an endomorphism $\tilde{\gamma}$ of $[a, b]$ such that $\tilde{\Psi}=\tilde{f} \circ \tilde{\gamma}$ is a continuous strictly increasing function. But then $\tilde{\Psi}(a)=c$ and $\tilde{\Psi}(b)=d$, and this implies that $\gamma=\tilde{\gamma}_{\mid(a, b)}$ is an endomorphism of $(a, b)$. Since $\tilde{f} \circ \gamma=f \circ \gamma$ on $(a, b)$ we get the conclusion.

Notice that in the above corollary we have again two possible complete rearrangements for the case of an open interval, but just one for the case of a half-open interval.

Now we investigate the case of unbounded connected sets.

Lemma 4.6. Let $X \subset \mathbb{R}$ be an open unbounded interval (resp. half-open unbounded interval). Then $f$ is completely rearrangeable if and only if $f(X)$ is a not necessarily unbounded open interval (resp. half-open interval), and

$$
0<\mu\left(f^{-1}([a, b])\right)<\infty, \quad \forall a, b \in f(I) \text { s.t. } a<b .
$$

Proof. We just prove the case $X=f(X)=\mathbb{R}$. (Along the same line, with simple modifications, the other cases follow.) The proof of necessity proceeds almost as before, and is left to the reader. So we assume that ( $\ddagger)$ holds. Write $\mathbb{R}=\bigcup_{n \in \mathbb{Z}}[n, n+1)$, and let $A_{n}=f^{-1}([n, n+1))$. Putting $J_{n}=\mu\left(A_{n}\right)$, from 
( $\ddagger$ ) we get that $0<J_{n}<\infty$. Let

$$
L_{n}= \begin{cases}\sum_{k=0}^{n} J_{k} & \text { if } n \geq 0 \\ 0 & \text { if } n=-1 \\ -\sum_{k=n+1}^{1} J_{k} & \text { if } n \leq-2,\end{cases}
$$

Applying Theorem 3.5, construct for every $n \in \mathbb{Z}$ an isomorphism $\beta_{n}$ between $A_{n}$ and the set $I_{n}=\left[L_{n-1}, L_{n}\right)$. Clearly, $\beta(x)=\beta_{n}(x)$ if $x \in A_{n}$ is an endomorphism of $\mathbb{R}$. Consider now $h=f \circ \beta^{-1}$. Observe that $h\left(I_{n}\right)=$ $[n, n+1)$. Thus, from Corollary 4.5, there exist isomorphisms $\alpha_{n}$ between $I_{n}$ and $[n, n+1)$, and continuous strictly increasing functions $\Psi_{n}: I_{n} \rightarrow[n, n+1)$ such that $h \circ \alpha_{n}=\Psi_{n}$ on $I_{n}$. Now setting $\Psi(x)=\Psi_{n}(x)$ if $x \in I_{n}$, and $\alpha(x)=\alpha_{n}(x)$ if $x \in I_{n}$, we get at once that $\Psi$ is a continuous strictly increasing function from $\mathbb{R}$ onto $\mathbb{R}$, and $\alpha$ is an endomorphism of $\mathbb{R}$. By construction $h \circ \alpha=\Psi$. Finally, from Remark 3.2, we conclude that $\gamma=\beta^{-1} \circ \alpha$ is a complete rearrangement of $f$.

We summarize our results in the following theorem.

Theorem 4.7. Let $X \subset \mathbb{R}$ be a connected set, and let $f: X \rightarrow \mathbb{R}$ be a oneto-one measurable function. Then $f$ is completely rearrangeable if and only if

(i) $X$ is a closed bounded interval and $f(X)$ is a closed bounded interval, or

(ii) $X$ is an open interval and $f(X)$ is an open interval, or

(iii) $X$ is an half-open interval and $f(X)$ is a half-open interval

(where we allow open and half-open intervals to be possibly unbounded), and the condition

$$
0<\mu\left(f^{-1}([a, b])\right)<\infty, \quad \forall a, b \in f(X) \text { s.t. } a<b
$$

holds.

We conclude the paper showing that rearrangeability and continuity almost everywhere, quite surprisingly, are not related concepts. Consider a function $f: X \subset \mathbb{R} \rightarrow \mathbb{R}$, with $X$ connected. With reference to [11], $f$ has $C$-limit $l$ at $x$ if for every $\varepsilon>0$ there exists $\delta>0$ such that

$$
\mu\left((x-\delta, x+\delta) \cap f^{-1}((\mathbb{R} \backslash(l-\varepsilon, l+\varepsilon)))=0 .\right.
$$


Note that it means that $l$ is the limit at $x$ in the ${ }^{*}$-topology of Hashimoto with respect to $\sigma$ ideal of null sets (see [5]). We denote by $\mathcal{C}_{f}$ the subset of $\mathbb{R}$ of the points where $f$ admits $C$-limit. The following theorem holds (see [11]).

Theorem 4.8. Given $f: X \subset \mathbb{R} \rightarrow \mathbb{R}$, there exists $g=f$ a.e. $(\mu)$ whose set of points of continuity is exactly $\mathcal{C}_{f}$.

It is a natural question to ask whether there is a relation between rearrangeability and continuity almost everywhere. Of course, we restrict our attention to Darboux functions (the Heaviside step function, for instance, is not rearrangeable, since its image is disconnected, but it is continuous everywhere except in the origin). In fact, even in this case, things go as wrong as possible. We will show that there exists a Darboux function $f$ on $[0,1]$, continuous almost everywhere and such that $\mathcal{C}_{f}=[0,1]$, which is not even rearrangeable. Conversely, we provide an example of a completely rearrangeable function $f$ such that $\mathcal{C}_{f}=\emptyset$.

Example 4.9. Let $X=[0,1]$, and consider the sequence $a_{n}=1-1 / n$, for $n \in \mathbb{N}$, and $a_{0}=1$. Define $f$ to be

$$
f(x)= \begin{cases}1 & \text { if } x=0, \\ x & \text { if } x>0 \text { and } x \neq a_{n}, n \in \mathbb{N}, \\ a_{n-1} & \text { if } x=a_{n}, n \in \mathbb{N} .\end{cases}
$$

Since $f(x)=x$ a.e. $(\mu)$, we have that $\mathcal{C}_{f}=[0,1]$. Moreover, $f$ is onto $[0,1]$. On the other hand, $\left|f^{-1}(\{x\})\right|=1$ for every $x \in[0,1)$, and $\left|f^{-1}(\{1\})\right|=2$. It is straightforward to see that no continuous function from $[0,1]$ onto $[0,1]$ can have all the fibers of cardinality 1 except one fiber of cardinality 2 . Thus $f$ is not rearrangeable.

Example 4.10. Let $X=[-1,1]$. It is well-known that it is possible to construct a set $T \subset(0,1)$ such that $0<\mu([a, b] \cap T)<b-a$ for every $0 \leq a<b \leq 1$.

Define $f$ by

$$
f(x)= \begin{cases}x & \text { if } x \in T \\ x-1 & \text { if } x \in[0,1] \backslash T \\ -x-1 & \text { if } x \in-T, \\ -x & \text { if } x \in[-1,0) \backslash-T\end{cases}
$$

It is easily verified that $\mathcal{C}_{f}=\emptyset$. On the other hand, $f$ is a bijection from $[-1,1]$ onto $[-1,1]$. Moreover for every $-1 \leq a<b \leq 1$,

$$
\mu\left(f^{-1}([a, b])\right) \geq \mu([a, b] \cap(-T \cup T))>0 .
$$


Hence, from Theorem 4.7, $f$ is completely rearrangeable.

\section{Acknowledgments}

The authors wish to thank Riccardo Re and Adele Zucchi for helpful discussion and comments. They also thank the referee for many valuable remarks and suggestions.

\section{References}

[1] P. Billingsley, Probability and measure, John Wiley \& Sons, New York, 1986.

[2] K. Ciesielski, R. Gibson, T. Natkaniec, $\kappa$-to-one Darboux-like functions, Real Analysis Exchange 23 (1997-98), 671-687.

[3] D. H. Fremlin, Measure Theory, vol.3, available on line.

[4] O. G. Harrold, The non-existence of a certain type of continuous transformation, Duke Math. J. 5 (1939), 789-793.

[5] H. Hashimoto, On the ${ }^{*}$-topology and its applications, Fund. Math. 91 (1976), 275-284.

[6] J. W. Heath, Every exactly 2-to-1 function on the reals has an infinite set of discontinuities, Proc. Amer. Math. Soc. 98 (1986), 369-373.

[7] J. W. Heath, There is no exactly $k$-to-1 function from any continuum onto $[0,1]$, or any dendrite, with only finitely many discontinuities, Trans. Amer. Math. Soc. 306 (1988), 293-305.

[8] E. Hewitt, K. Stromberg, Real and abstract analysis, Springer-Verlag, New York, 1965.

[9] H. Katsuura, The nonexistence of a continuous surjection from a continuum onto its square, Proc. Amer. Math. Soc. 111 (1991), 1129-1140.

[10] H. Katsuura, K. R. Kellum, k-to-1 functions on an arc. Proc. Amer. Math. Soc. 101 (1987), 629-633.

[11] V. Pata, Lebesgue measurability and continuity of real functions, submitted.

[12] V. A. Rohlin, On the fundamental ideas of measure theory, Amer. Math. Soc. Translation 71, 1952. 